\title{
Choosing a model for recruiting 100,000 pregnant women to the Norwegian Mother and Child Cohort Study (MoBa)
}

\author{
Kjell Haug ${ }^{1}$ and Patricia Schreuder ${ }^{2}$ \\ 1) University of Bergen, Bergen, Norway \\ 2) National Institute of Public Health, Bergen, Norway
}

This is an open access article distributed under the Creative Commons Attribution Licence, which permits unrestricted use, distribution, and reproduction in any medium, provided the original work is properly cited.

From a methodological point of view, it was important to recruit women to $\mathrm{MoBa}$ as early as possible after they had become pregnant. We wanted mothers to fill in a questionnaire and give a blood sample early in the first trimester. In that way, we would have information from the mother about her lifestyle, occupation, social status, use of medication and dietary supplements and much more as well as a biological sample at the beginning of the pregnancy and we could follow the pregnancy through the next questionnaires and another blood sample late in pregnancy or at birth. We would continue to follow the mother and child through infancy and childhood to adulthood.

We decided on a model for recruitment where women were recruited when they consulted their general practitioner for the first check-up in their pregnancy. This consultation most often takes place 6-10 weeks after their last menstrual period. Therefore, in September 1995, we invited all general practitioners in two municipalities outside Bergen (Fjell and Sund with a total population of about 30,000 ) to an information meeting. The response was very positive. The GPs were enthusiastic. From January 1996 until the end of January 1997, over 240 pregnant women were invited to participate in the pilot. The participation rate was high $-80 \%$ decided to participate.

Our plan was to expand the study to incorporate Hordaland County with a population of about 400,000 and then gradually extend to the whole of Norway. The population of Norway at this time was about 4,500,000 with approximately 60,000 births per year. In 1996 there were 300 general practitioners in Hordaland County and more than 3,000 in Norway. A great number of general practitioners would have to be involved in order to recruit all pregnant women in Norway to MoBa. If we were optimistic, and if the participation rate of $80 \%$ in the pilot study was consistent for the whole country, we would have 100,000 participating women within two years. However, we realized that to establish good cooperation with so many GPs would be quite complicated and very challenging. In addition, we would have to work together with a large number of municipal midwives since pregnant women also consult midwives during pregnancy.

During the autumn of 1996 a debate was started among general practitioners in Norway regarding ethical aspects of the study. Some influential GPs argued against the study and were very active in their criti- cism in medical journals, newspapers and other fora. They claimed that participating in the study and answering the questionnaires could cause anxiety among pregnant women. It would be time consuming for GPs during a busy consultation and GPs would have no financial compensation for the increased work load resulting from giving out information and trying to recruit to the study. Being such an ambitious and expensive study, MoBa could threaten financial support to other potential studies which might be more interesting for some GPs. Even the name of the study "Better Health for Mother and Child" was problematic. Criticism of the project became so serious that it was debated in the Norwegian Parliament. The Government decided that the project should be stopped. A committee was appointed to conduct an inquiry. The committee's report would advise Parliament whether or not the project should be allowed to continue with its plans. Permission to continue was granted in June 1998, but with little financial support.

At this time, the MoBa study group started to investigate a new model for recruitment should permission be granted and the study became national. We realized that cooperation over a number of years with several thousand more or less motivated GPs would be complicated and expensive and might be very difficult to achieve.

An alternative model was to recruit pregnant women when they attended their ultrasound examination at the local hospital around week 17 in the pregnancy. 98\% of all pregnant women attend ultrasound screening. Cooperation with the 52 hospitals with maternity units in Norway seemed a more realistic and affordable task. We also realized that the ultrasound examination could be a "golden opportunity" for recruitment at a time when mothers, and even fathers, were highly motivated. Being in the second trimester also meant that women who had decided to terminate the pregnancy or who had had a spontaneous abortion would not be invited to participate. Midwives and gynecologists at the ultrasound examinations were also probably more likely to be enthusiastic about such a study. It was therefore decided that, given permission to restart the project and begin recruitment, this alternative would be preferable.

Pregnant women would be sent an invitation to participate in the study in connection with their appointment for ultrasound examination at the local 
hospital. The invitation would include information about the study, an informed consent form, a questionnaire and information explaining why it was necessary to have a blood sample from the mother and the father, when later in the study fathers were also invited.

In order to organize and recruit to the study at the larger hospitals, it was necessary to employ midwives who were dedicated to MoBa.
The biggest drawback with the ultrasound model compared with the GP-model was the time for recruitment. The ultrasound model meant that pregnant women were 10 weeks later in their pregnancy when they filled in the questionnaire and gave their bloodsample. Except from this disadvantage, the ultrasound model was less expensive and easier to manage. 